\title{
Dosimetric Comparative Study of Static Gantry IMRT and 3D Conformal Radiotherapy for Organ at Risk in Adjuvant Therapy of Gastric Cancer
}

\author{
Hanan S. Darwish \\ Clinical Oncology Department, Kasr Al-Ainy Center of Clinical Oncology and Nuclear Medicine; \\ Kasr Al-Ainy School of Medicine, Cairo University, Cairo, Egypt.
}

Background: Despite the benefits of postoperative radiotherapy for gastric cancer patients after publishing the results of the Intergroup 0116 (INT-0116) study, there are still the concerns of radiation-induced toxicity.

Aim: To determine the radiation technique that can eliminate the dose of radiation to the organ at risk (OAR) in patients postoperatively treated for gastric cancer with three-dimensional conformal radiotherapy (3D-CRT) or intensitymodulated radiotherapy (IMRT) using dosimetric analysis.

Methods: A total of 22 computerized radiation plans were reviewed. Dosimetric values for 3DCRT and IMRT technique were calculated. The following dosimetric parameters were compared for the planned target volume (PTV); the homogeneity index, maximal and minimal doses, the volumes of PTV received $95 \%$ and $107 \%$ of the prescribed dose. In addition, the mean dose and dose volume histogram (DVH) for the OAR as, V20 for each kidney, V30 for the liver, maximum dose (Dmax) for D1\% of the spinal cord and V40 to the heart .

Results: The 3D-CRT and IMRT achieved comparable PTV coverage. However, IMRT was associated with a highly significant decrease in the mean V20 value of the right kidney by $15 \%(\mathrm{P}=0.001)$ and left kidney by $9 \%(\mathrm{P}=0.01)$. The IMRT significantly improved sparing of the mean irradiated volume and the V30 of the liver by $12 \%(\mathrm{P}=0.01)$ and by $10 \%(\mathrm{P}=0.02)$ respectively compared with 3D-CRT. On the other hand, the dosimetric parameters for the spinal cord and heart were comparable for the IMRT and 3D-CRT plans.

Conclusion: In gastric cancer patients receiving postoperative radiotherapy, IMRT provided better protection for the liver and kidneys when compared to 3D-CRT.

Keywords: Dosimetric, Three-dimensional conformal radiotherapy (3D-CRT), Intensity- modulated radiotherapy (IMRT), Gastric cancer

Corresponding author: Hanan Darwish, MD; Clinical Oncology Department, Kasr Al-Ainy Center of Clinical Oncology and Nuclear Medicine; Kasr Al-Ainy School of Medicine, Cairo University, Cairo, Egypt; Email: hanan.darwish@kasralainy.edu.eg

Submitted: 5-Mar-2017, Revised: 28-April-2017, Accepted: 29-Apr-2017

\section{INTRODUCTION}

As per population-based cancer registries in Egypt, gastric cancer (GC) is the 12 th most common cancer in both sexes. It represents $1.6 \%$ of all malignancies and $2.2 \%$ of the total Egyptian cancer mortality ${ }^{1}$. As per expectation, Egypt will report 1249 new cases of gastric cancer by 2020 . The incidence varies within different geographic areas in Egypt. The highest incidence was reported in Southern Upper Egypt $(2.48 \%)$ and the lowest in Northern governorates $(0.98 \%)^{2}$. Locoregional recurrence is a significant problem with a reported rate of $23 \%$ to $38 \%$, emphasizing the need for adjuvant local therapy ${ }^{3}$. However, several studies demonstrated a favorable outcome with adjuvant chemoradiotherapy for operable cases ${ }^{4-6}$. Since the target volume has to be large and often not be irradiated in its entity to a sufficient dose, the toxicity of adjuvant chemo-radiotherapy is high ${ }^{7}$. Therefore, conformal radiotherapy have been used to overcome the drawbacks of conventional techniques, such as under-dosage of target regions and high radiation to surrounding normal structures. However, there is debate which conformal technique (three-dimensional conformal radiotherapy [3D-CRT] or intensity- modulated radiotherapy [IMRT]) is better for gastric cancer radiotherapy ${ }^{8,9}$.

This study was conducted to determine which radiation technique can eliminate the dose of radiation to the organ at risk (OAR) using retrospective dosimeter analysis among gastric cancer patients treated with postoperative 3D-CRT or IMRT, at the Center of Clinical Oncology and Nuclear Medicine.

\section{METHODS}

This is a retrospective review of computerized radiation plans of 22 patients diagnosed with nonmetastatic gastric adenocarcinoma. Patients received the postoperative radiotherapy at the Center of Clinical Oncology and Nuclear Medicine, Kasr Al-Ainy School of Medicine, Cairo University, Egypt. We used 3D-CRT in 9 patients (40.9\%) and IMRT in $13(59.1 \%)$.

\section{Radiation Therapy}

For adjuvant post-operative patients, free breathing scans were used with $1-\mathrm{cm}$ CT slices. The clinical target 
volume (CTV) was defined from the pre-operative endoscopy, CT scan and operative details and clips. It included preoperative tumor extension, gastric bed (for total gastrectomy) or remnant (for subtotal gastrectomy), site of anastomoses and the draining lymph node: paraaortic and para-caval, celiac axis, hepatic artery, and splenic artery. For proximal lesions, the medial twothirds of the left hemi-diaphragm and the lower paraesophageal nodes were included in the CTV.

For setup variation and organ motion, the CTV was expanding with $1 \mathrm{~cm}$ in all directions for construct the planning target volume (PTV) ${ }^{10}$. The OAR contouring included the liver, both kidney, spinal cord, heart, and lungs. The prescribed dose varied between 45-50.4 Gy in 25-28 fractions. The dose constraints for the OAR included: the volume of the contralateral kidney exposed to $>20 \mathrm{~Gy}(\mathrm{~V} 20)$ was $<30 \%$, the mean dose (Dmean) for each kidney was $<18 \mathrm{~Gy}$, the percentage of normal liver volume that was irradiated with $>30$ Gy (V30) was $<30 \%$, the maximum spinal cord dose $<45$ Gy and $<30 \%$ of the volume of cardiac silhouette exposure to a dose of 40 Gy (V40) ${ }^{10,11}$.

All plans were generated for a Xio workstation version 4.8 module (USA). The plan for 3D-CRT was either 3 or 4 field-technique and 5-7-fields for IMRT. Radiation treatment was delivered with a 6-MV linear accelerator. IMRT was delivered with a step-and-shoot technique.

To evaluate target coverage, the following dosimetric parameters were compared: the volumes received the minimal, maximal, $95 \%$ (V95\%) and 107 $\%(\mathrm{~V} 107 \%)$ of the prescribed dose for PTV. For evaluating plan quality, the homogeneity index (HI) was calculated as $\mathrm{HI}=\mathrm{Imax} / \mathrm{RI}$; where Imax is the maximum dose to the target, and RI is the reference dose in the PTV. HI values far from 1 are the poorer the dosedistribution uniformity. In addition, we analyzed and compared the dosimetric parameters for OAR as follows: the Dmean (mean dose) and the partial volume doses that received 20 Gy (V20) for each kidney, V30 for the liver, maximum dose (Dmax) for D1\% of the spinal cord and V40 of the heart. The small intestine has a tendency to shift between fractions and lung was frequently not delineated in many cases, so we did not include these organs in the evaluation of OAR.

\section{Statistical analysis}

The dosimetric parameters were analyzed using SPSS software version 22 (SPSS, Inc., Chicago, IL, USA) and all parameters were presented as the mean \pm standard deviation. Dosimetric parameters of both techniques were compared by the paired samples t-test. Statistically significant differences imply a p-value of < 0.05 .

\section{RESULTS}

\section{Criteria of PTV coverage}

The median volume of the PTV was $1349.4 \mathrm{~cm}^{3}$ (Range; 958.70 - 2362.3) for IMRT versus $1578.2 \mathrm{~cm}^{3}$ (Range; 900 - 2694.9) for 3D-CRT technique ( $\mathrm{P}=0.4)$. The average minimum dose in the PTV was $41.71 \mathrm{~Gy}$ for 3D-CRT versus $42.45 \mathrm{~Gy}$ for IMRT $(\mathrm{P}=0.65)$. The Dmax of 3D-CRT plan in PTV was higher than that in IMRT plan (50.37 vs $48.66 \mathrm{~Gy}$; respectively) but with no statistically significant difference $(\mathrm{P}=0.07)$. Additionally, there was no significant differences in the Dmean between IMRT and 3D-CRT (44.26 vs 44.35 GY $\mathrm{P}=0.97)$. Similarly, the median volume received $107 \%$ of prescribed dose was $1.9 \%(0.9-2.8 \%)$ in comparison to $2 \%(0.2-3)$ by $3 \mathrm{D}-\mathrm{CRT}$ techniques $(\mathrm{P}=0.7)$. Regarding target uniformity, IMRT plans was superior in HI (1.04 $\pm 0.02)$ in the PTV compared to the 3D-CRT plan (1.06 $\pm 0.03)$ with no significance $(\mathrm{P}=0.43)$ as shown in Table 1. These volumes were not significantly different indicating that PTV coverage was comparable between both techniques. Figures (1 and 2) show examples of isodose curves for 3D-CRT and IMRT plans, respectively.

\section{Criteria of OAR dosimetry}

Spinal parameters: Values of the mean volume irradiated and Dmean as well as the Dmax for spinal cord in 3D-CRT $\left(50.9 \mathrm{~cm}^{3}, 18.83 \mathrm{~Gy}\right.$ and 30.13Gy, respectively) were nearly similar to the values in IMRT technique $(49.1 \mathrm{~cm} 3,21.18 \mathrm{~Gy}$ and 28.46Gy respectively) and the differences were not significant $(\mathrm{P}$ $>0.05)$

Renal parameters: Compared to 3D-CRT, IMRT reduced the mean volume included in the PTV (181 vs $215 \mathrm{~cm}^{3}, \mathrm{P}=0.5$ ) of the right kidney but the difference was not significant. Despite a highly significant lower meanV20 value of the right kidney in the IMRT technique by $15 \%$ ( $19 \%$ vs $34 \%$; $\mathrm{P}=0.001)$, the Dmean (18.52 vs 18. $97 \mathrm{~Gy}, \mathrm{P}=0.9$ ) were not significantly lowered, this may be due to a large volume irradiated with low dose. IMRT significantly reduced the V20 volume of left kidney by $9 \%$ when compared with 3DCRT planning ( $42.5 \%$ vs $33.5 \%$, respectively; $\mathrm{P}=0.01)$. Also, the mean volume of the left kidney included in the PTV was reduced in IMRT planning (230.4 vs $683.1 \mathrm{~cm}^{3}$ ) but with no statistically significant difference $(\mathrm{P}=0.2)$. The Dmean of the left kidney was similar and not exceeded the tolerance dose in both techniques $3 \mathrm{D}$ CRT and IMRT (17.14 vs17.32 Gy; $\mathrm{P}=0.9$ ). The mean dose values received by both kidneys are summarized in Table 2.

Table 1: Dose-volume histogram parameters of the planning tumor volume

\begin{tabular}{|c|c|c|c|}
\hline Parameters & $\begin{array}{l}\text { 3D-CRT } \\
(\mathrm{n}=9)\end{array}$ & $\begin{array}{l}\text { IMRT } \\
(n=13)\end{array}$ & $\begin{array}{l}\text { P- } \\
\text { value }\end{array}$ \\
\hline \multicolumn{4}{|c|}{ Mean \pm SD } \\
\hline Volume $\left(\mathrm{cm}^{3}\right)$ & 1578.2 & 1349.4 & 0.41 \\
\hline Minimum dose (Gy) & $41.71 \pm 82$ & $42.45 \pm 44$ & 0.65 \\
\hline Maximum dose (Gy) & $50.37 \pm 23$ & $48.66 \pm 28$ & 0.23 \\
\hline V95\% & $\begin{array}{l}96.82 \% \pm \\
0.51\end{array}$ & $\begin{array}{l}97.91 \% \pm \\
0.3\end{array}$ & 0.01 \\
\hline $\mathrm{V} 107 \%$ & $2 \%$ & $1.9 \%$ & 0.71 \\
\hline Homogeneity index & $1.06 \pm 0.03$ & $1.04 \pm 0.02$ & 0.43 \\
\hline
\end{tabular}




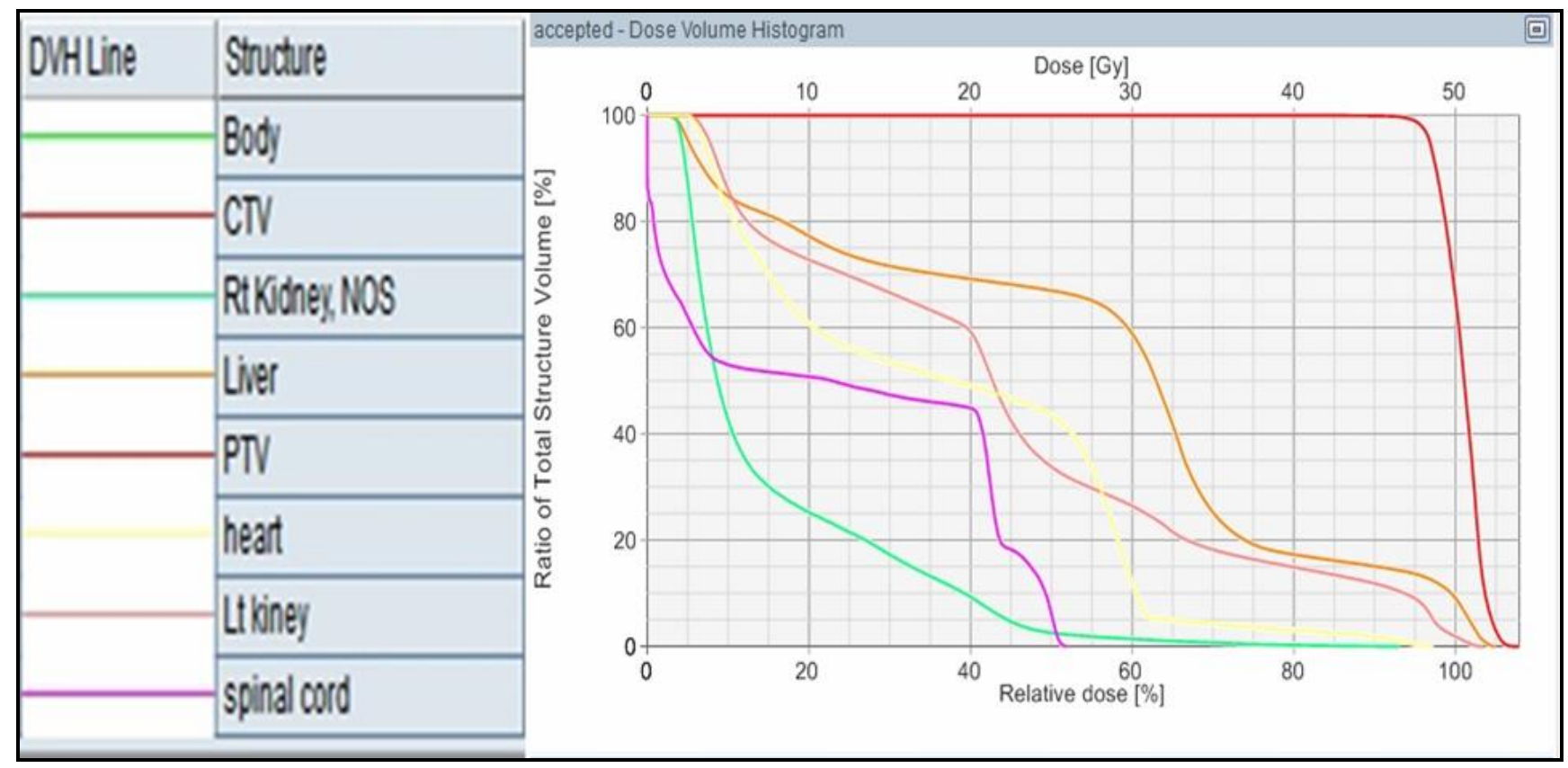

Figure 1: Dose volume histogram (DVH) parameters of 3D-CRT

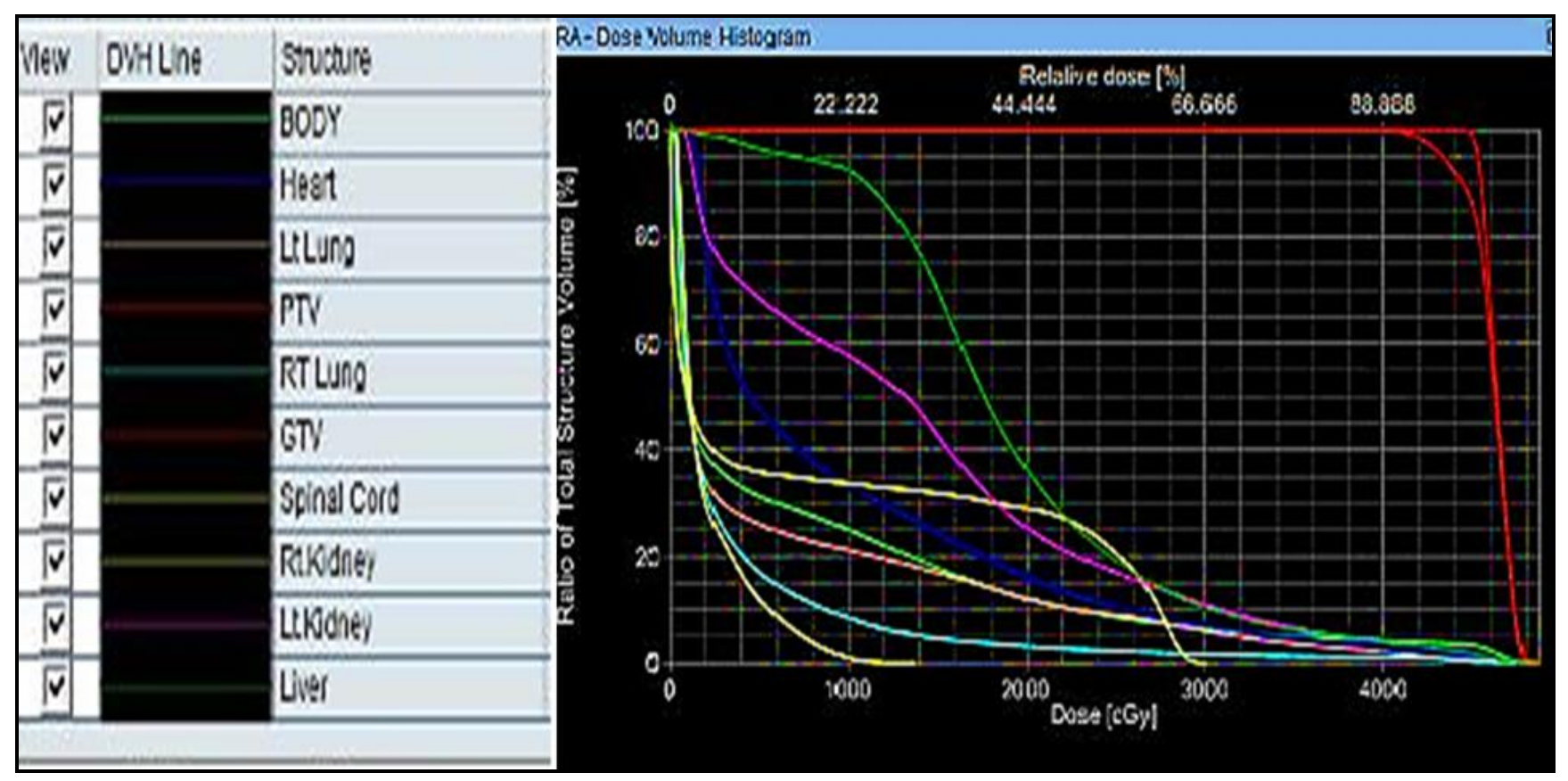

Figure 2: Dose volume histogram (DVH) parameters of IMRT

Liver parameters: IMRT significantly decreased the mean volume $\left(1537.3\right.$ vs $\left.1889.7 \mathrm{~cm}^{3} ; \mathrm{P}=0.01\right)$ and the mean $\mathrm{V} 30(21 \%$ vs $31 \%$; $\mathrm{P}=0.02)$ of the liver in compare to 3D-CRT. Furthermore, by IMRT technique, the Dmean (22.85 vs 23.61 Gy) was reduced but with no significant difference $(\mathrm{P}=0.6)$.

Heart parameters: No statistically significant trends for both techniques as regard the average volume $(\mathrm{P}=0.54)$, Dmean $(\mathrm{P}=0.53)$, Dmax $(\mathrm{P}=0.99)$ and $\mathrm{V} 30$ $(\mathrm{P}=0.64)$ for the heart.

Table 3 summarizes the DVH parameters for the spinal cord, liver and the heart.

\section{DISCUSSION}

Postoperative chemo-radiation has become the standard of care for patients with gastric cancer after the publication of Intergroup 0116 trial ${ }^{7}$. However, due to the large irregular target area to cover and the proximity of many vital organs to this region, it was difficult to achieve satisfactory dose distribution with 3D-CRT, so treatment interruption was documented in $25 \%$ patients because of the toxicity ${ }^{12,13}$. 
Table 2: Dose-volume histogram (DVH) parameters of both kidneys

\begin{tabular}{|c|c|c|c|c|c|}
\hline Variables & & & 3D-CRT $(n=9)$ & IMRT $(\mathrm{n}=13)$ & P-value \\
\hline \multicolumn{6}{|c|}{ Right kidney } \\
\hline & \multirow[t]{2}{*}{ Volume $\left(\mathrm{cm}^{3}\right)$} & Mean & 215 & 181 & \multirow[t]{2}{*}{0.5} \\
\hline & & Range & $84.6-256$ & $71.2-712$ & \\
\hline & \multirow[t]{2}{*}{ Dose (Gy) } & Dmean & 18.52 & 18.97 & 0.9 \\
\hline & & $\mathrm{V} 20$ & $34 \%$ & $19 \%$ & 0.001 \\
\hline \multicolumn{6}{|l|}{ Left kidney } \\
\hline & \multirow[t]{2}{*}{ Volume $\left(\mathrm{cm}^{3}\right)$} & Mean & 683.1 & 230.4 & \multirow[t]{2}{*}{0.2} \\
\hline & & Range & $95.3-276.7$ & $80.1-811$ & \\
\hline & \multirow[t]{2}{*}{ Dose (Gy) } & Dmean & 17.14 & 17.32 & 0.9 \\
\hline & & V20 & $43 \%$ & $34 \%$ & 0.01 \\
\hline
\end{tabular}

3D-CRT: 3-dimensional conformal radiotherapy; IMRT: intensity-modulated radiotherapy; Dmean: the mean dose; V20: percent of volume received 20 Gy

Table 3: DVH parameters of 3D-CRT and IMRT for the organs at risk

\begin{tabular}{|c|c|c|c|c|}
\hline \multicolumn{2}{|l|}{ Organ at risk parameters } & 3D-CRT $(n=9)$ & IMRT $(\mathrm{n}=13)$ & P-value \\
\hline \multicolumn{5}{|l|}{ Spinal cord } \\
\hline \multirow[t]{2}{*}{ Volume $\left(\mathrm{cm}^{3}\right)$} & Mean & $50.9 \pm 19$ & $46.4 \pm 20$ & \multirow[t]{2}{*}{0.7} \\
\hline & Range & $24.7-73.6$ & $28.3-79.5$ & \\
\hline Dose (Gy) & Maximum & 30.13 & 28.46 & 0.7 \\
\hline \multicolumn{5}{|l|}{ liver } \\
\hline \multirow[t]{3}{*}{ Volume $\left(\mathrm{cm}^{3}\right)$} & Mean & $1889.7 \pm 19$ & $1537.3 \pm 28$ & \multirow[t]{2}{*}{0.01} \\
\hline & Range & $1650-2184$ & $670-1995.1$ & \\
\hline & $\mathrm{V} 30$ & $31 \%$ & $21 \%$ & 0.02 \\
\hline Dose (Gy) & Mean & 23.61 & 22.85 & 0.6 \\
\hline \multicolumn{5}{|l|}{ Heart } \\
\hline \multirow[t]{2}{*}{ Volume $\left(\mathrm{cm}^{3}\right)$} & Mean & 555 & 501 & \multirow[t]{2}{*}{0.54} \\
\hline & Range & $325-896$ & $301-885$ & \\
\hline \multirow[t]{2}{*}{ Dose (Gy) } & Mean & 23.58 & 21.23 & \\
\hline & V40 & $15 \%$ & $17 \%$ & 0.64 \\
\hline
\end{tabular}

DVH: Dose-volume histogram; 3D-CRT: 3 -dimensional conformal radiotherapy; IMRT: intensity-modulated radiotherapy.

Theoretically, IMRT can reduce the upper GI radiation toxicity. Therefore, several dosimetric studies compared 3D-CRT to IMRT in adjuvant gastric cancer radiotherapy and reported that IMRT improved the sparing of critical structures ${ }^{14-16}$. Two published studies, from the University of Heidelberg, compared the dose distribution of different planning modalities, and observed reduction of the median dose to the left kidney and the doses to V30 and V60 to the kidneys and liver by IMRT ${ }^{17-19}$. Some investigators reported that the clinical gains yielded by IMRT are not superior to 3DCRT in terms of OS, RFS and toxicity in gastric cancer patients ${ }^{20-23}$.

The rationale of the present study is to determine, on a planning basis, which radiation techniques can eliminate the delivering radiation dose to OAR. Our results demonstrate that, IMRT was superior to 3D-CRT for target volume homogeneity index and reduced the target maximum dose and hot spots, but did not show a statistically significant advantage over 3D-CRT. Our results were consistent with two recent studies by Zhang et al and Chopra et al ${ }^{24,25}$. Zhang et al compared the dose distributions of different radiation techniques as adjuvant radiotherapy modalities for 15 patients with gastric carcinoma and observed no significant difference in the maximum, minimum doses and the V95\% target volume between the 3D-CRT and IMR; however, IMRT was superior in the PTV uniformity compared to 3DCRT $(\mathrm{P}<0.05){ }^{24}$. In the second study, Chopra et al. reported similar median radiation dose to the PTV in the both plans. Moreover, IMRT significantly reduced the dose received by both kidney $(\mathrm{P}=0.001)^{25}$.

The Dmean and V20 are the most DVH parameters that predict the renal toxicity ${ }^{26-28}$. Therefore, in the current study, V20 and Dmean parameters were selected to evaluate the irradiated kidneys. The most important finding in our research is that, IMRT had significantly lowered the meanV20 value of the right kidney by $15 \%$ $(\mathrm{P}=0.001)$ and of the left kidney by $9 \%(\mathrm{P}=0.01)$, which was consistent to previous retrospective studies $18,19,21$. In addition, we observed the decrease of the mean irradiated volume of both kidneys by IMRT technique but such decrease was not significant, subsequently; the Dmean values were similar in both techniques. Minn et al compared the toxicity in gastric cancer patients treated with postoperative chemo-radiotherapy using IMRT versus 3D-CRT, and observed a significant increase in the median serum creatinine levels in patients treated with 3D-CRT $(\mathrm{P}=0.02)^{14}$.

The incidence of radiation-induced liver disease (RILD) is significantly correlated to the mean dose to normal liver (MDTNL). Dawson et al reported that no RILD was detected when the mean liver dose was <31 Gy where, each 1-Gy-increase in MDTNL, increased the incidence of RILD by $4 \%{ }^{29}$. Meanwhile, Liang et al demonstrated that when the MDTNL was $23 \mathrm{~Gy}$, the RILD occurrence rate was $6 \%$. Therefore, a MDTNL of 23 Gy may be used as a predictor of RILD ${ }^{30}$. 
Noteworthy, the risk of reactivation of hepatitis B virus $(\mathrm{HBV})$ by radiation has been observed ${ }^{31}$. Special measurements should be considered for such subgroup of patients through sparing the liver volume and limit the dosage of radiation to the normal hepatic tissues by using high precise technology, as well as monitoring of liver function and routine investigation of the HBV-DNA copy numbers ${ }^{32}$.

Dosimetric parameters, such as the mean liver dose and $\mathrm{V} 30$, are predictors of increased toxicity risk during partial liver radiation 30,33 . Therefore, in our study, we selected Dmean and V30 parameters to estimate the dose distribution in liver. The used liver dose in this work seems to be acceptable and does not exceed the aforementioned dose limit requirements. IMRT significantly improved sparing of the mean irradiated volume of the liver by $12 \%(\mathrm{P}=0.01)$ and the meanV30 by $10 \%(\mathrm{P}=0.02)$ compared to 3D-CRT. Conversely, we observed a little difference between IMRT and 3D-CRT techniques as regard of the Dmean $(\mathrm{P}=0.6)$ which may be due to low dose irradiation to a large volume.

Radiation-induced heart disease occurs when the heart receives more than $40 \mathrm{~Gy}{ }^{32}$. Our results showed that lower heart dose delivered by both modalities that reducing the chances of late cardio-related toxicity to develop.

High radiation dose can result in radiation-induced spinal myelitis. The incidence of spinal myelitis was found to be $<0.2 \%$ at a dose $45 \mathrm{~Gy} / 25$ fractions ${ }^{34}$. In our study, the spinal Dmax was higher in 3D-CRT when compared to IMRT technique (30.13 vs 28.46 Gy; respectively) with no statistical difference $(\mathrm{P}=0.76)$.

\section{Conclusions}

On the basis of treatment plan comparison, the present study observed the clear advantage of IMRT to liver and kidney sparing over 3D-CRT in the adjuvant radiotherapy setting in gastric cancer, however some important issues need to be addressed.

Our results based on retrospective data of a small patient number, therefore, further trials may be required with a large number of patients in a prospective design to correlate the DVH parameters with liver and renal function tests to reduce radiation- induced toxicity.

\section{REFERENCES}

1. The National Cancer Registry Program of Egypt (NCRPE). Reports and Statistics: Aswan, Damietta \& ElMinia [Internet]. [Cited $2014 \mathrm{Feb} 22$ ].Available from :<http: //www.cancerregistry.gov.eg/oops.aspx ? aspxerrorpath=/ publications.aspx>

2. Gharbiah Population-Based Cancer Registry, Egypt, Cancer in Egypt, triennial report of 2000-2002. 2007. Ministry of Health and Population (MOH): Tanta, Egypt

3. Gunderson LL. Gastric cancer-patterns of relapse after surgical resection. Semin Radiat Oncol. 2002; 12(2): 150161.

4. Macdonald JS, Smalley S, Benedetti J, et al. Postoperative combined radiation and chemotherapy improves disease free survival (DFS) and overall survival (OS) in resected adenocarcinoma of the stomach and gastroesophageal junction: Update of the results of Intergroup Study INT-
0116 (SWOG 9008), Proceedings of the ASCO gastrointestinal cancers symposium 6; 2004.

5. Smalley SR, Benedetti JK, Haller DG, et al. Updated analysis of SWOG-directed Intergroup Study 0116: A phase III trial of adjuvant radio-chemotherapy versus observation after curative gastric cancer resection. J Clin Oncol. 2012; 30(19): 2327-2333.

6. Zhu WG, Xua DF, Pu J,et al. A randomized, controlled, multicenter study comparing intensity-modulated radiotherapy plus concurrent chemotherapy with chemotherapy alone in gastric cancer patients with D2 resection. Radiother Oncol. 2012; 104(3): 361- 366.

7. Macdonald JS, Smalley SR, Benedetti J, et al. Chemoradiotherapy after surgery compared with surgery alone for adenocarcinoma of the stomach or gastroesophageal junction. N Engl J Med. 2001; 345(10): 725-730.

8. Milano MT, Garofalo MC, Chmura SJ, et al. Intensitymodulated radiation therapy in the treatment of gastric cancer: early clinical outcome and dosimetric comparison with conventional techniques. Br J Radiol. 2006; 79(942): 497-503.

9. Alani S, Soyfer V, Strauss N, Schifter D, Corn BW. Limited advantages of intensity-modulated radiotherapy over $3 \mathrm{D}$ conformal radiation therapy in the adjuvant management of gastric cancer. Int $\mathbf{J}$ Radiat Oncol Biol Phys. 2009; 74(2): 562-566.

10. Matzinger O, Gerber E, Bernstein Z, et al. EORTC-ROG expert opinion: radiotherapy volume and treatment guidelines for neoadjuvant radiation of adenocarcinomas of thengastroesophageal junction and the stomach. Radiother Oncol. 2009; 92(2) : :164-175.

11. Dawson LA, Kavanagh BD, Paulino AC, et al. Radiationassociated kidney injury. Int J Radiat Oncol Biol Phys. 2010; 76(3 Suppl): S108-115.

12. Ringash J, Khaksary SJ, Oza A, et al. Post-operative Radiochemotherapy for Gastric Cancer: Adoption and Adaptation J Clin Oncol. 2005; 17: 91-95.

13. Zahra K, Gina L, Catherine O'Brien, et al. Conformal radiotherapy in the adjuvant therapy of gastric cancer: review of 82 cases. Int J Radiat Oncol Biol Phys. 2006; 65: 713-719.

14. Minn AY, Hsu A, La T, et al: Comparison of intensity- modulated radiotherapy and 3-dimensional conformal radiotherapy as adjuvant therapy for gastric cancer. Cancer. 2010; 116: 3943-3952.

15. Dahele M, Skinner M, Schultz B,et al. Adjuvant radiotherapy for gastric cancer: A dosimetric comparison of 3-dimensional conformal radiotherapy, tomotherapy and conventional intensity modulated radiotherapy treatment plans. Med Dosim. 2010; 35: 115-121.

16. Tang $\mathrm{H}$, Luo J, Lu X, et al. Dosimetry analysis of three kinds of radiation technique for postoperative gastric cancer. Advanced Materials Research. 2013; 641-642: 725-731.

17. Lohr F, Dobler B, Mai S, et al. Optimization of dose distributions for adjuvant locoregional radiotherapy of gastric cancer by IMRT. Strahlenther Onkol. 2003; 179: 557-563.

18. Wieland $\mathrm{P}$, Dobler $\mathrm{B}$, Mai $\mathrm{S}$, et al. IMRT for postoperative treatment of gastric cancer: covering large target volumes in the upper abdomen: a comparison of a step-and-shoot and an arc therapy approach. Int J Radiat Oncol Biol Phys. 2004; 59: 1236-1244.

19. Minn A, Fisher G, Ford J, et al. Comparison of IMRT and 3D Conformal Radiotherapy for Adjuvant Therapy for Gastric Cancer. Int J Radiat Oncol Biol Phys. 2009; 75(3, Suppl): S259. 
20. Shlomo A, Viacheslav S, Natan S,et al. Limited advantages of intensity modulated radiotherapy over 3D conformal radiotherapy in the adjuvant management of gastric cancer. Int J Radiat Oncol Biol Phys. 2009; 74(2): 562-566.

21. Boda-Heggemann J, Hofheinz RD, Weiss C, et al. Combined adjuvant radiochemotherapy with IMRT/XELOX improves outcome with low renal toxicity in gastric cancer. Int J Radiat Oncol Biol Phys. 2009; 75: $1187-1195$.

22. Liu GF, Bair RJ, Bair E, et al. Clinical outcomes for gastric cancer following adjuvant chemo-radiation utilizing intensity modulated versus three- dimensional conformal radiotherapy. PLoS One. 2014; 9: e 82642.

23. Minn AY, Hsu A, La T, et al. Comparison of intensitymodulated radiotherapy and 3-dimensional conformal radiotherapy as adjuvant therapy for gastric cancer. Cancer. 2010; 116: 3943-3952.

24. Zhang T, Zhi-Wen L, Jun H. Double-arc volumetric modulated therapy improves dose distribution compared to static gantry IMRT and 3D conformal radiotherapy for adjuvant therapy of gastric cancer. Radiation Oncology. 2015; 10:114.

25. Chopra S, Agarwal A, Engineer R. et al. Intensity modulated radiation therapy (IMRT) is not superior to three-dimensional conformal radiation (3DCRT) for adjuvant gastric radiation: A matched pair analysis. J Cancer Res Ther. 2015; 11(3): 623-629.

26. Chung HT, Lee B, Park E, et al. Can all centers plan intensity-modulated radiotherapy (IMRT) effectively? An external audit of dosimetric comparisons between threedimensional conformal radiotherapy and IMRT for adjuvant chemoradiation for gastric cancer. Int $\mathrm{J}$ Radiat Oncol Biol Phys. 2008; 71: 1167-1174

27. Lohr F, Dobler B, Mai S, et al. Optimization of dose distributions for adjuvant locoregional radiotherapy of gastric cancer by IMRT. Strahlenther Onkol. 2003; 179: 557-563.

28. Cassady JR. Clinical radiation nephropathy. Int J Radiat Oncol Biol Phys. 1995; 31: 1249-1256.

29. Dawson LA, Normolle D, Balter JM, et al. Analysis of radiation-induced liver disease using the Lyman NTCP model. Int J Radiat Oncol Biol Phys. 2002; 53: 810-821.

30. Liang SX, Zhu XD, Xu ZY, et al: Radiation-induced liver disease in three-dimensional conformal radiation therapy for primary liver carcinoma: the risk factors and hepatic radiation tolerance. Int J Radiat Oncol Biol Phys. 2006; 65: 426-434.

31. $\mathrm{Xu} \mathrm{J,} \mathrm{Zhu} \mathrm{H,} \mathrm{Zhao} \mathrm{Y,} \mathrm{et} \mathrm{al:} \mathrm{Factors} \mathrm{associated} \mathrm{with}$ hepatic dysfunction in hepatitis B-positive patients with postgastrectomy adenocarcinoma. Oncol Lett. 2012; 4: 471-476.

32. Zhiping L, Jianshuang Z, Wangi Z, et al. Dosimetric comparison of intensity modulated and volumetric arc radiation therapy for gastric cancer. Oncol Lett. 2014; 8: 1427-1434

33. Kim T, Kim D, Park J, et al. Dose-volumetric parameters predicting radiation induced hepatic toxicity in unresectable hepatocellular carcinoma patients treated with three-dimensional conformal radiotherapy. Int $\mathrm{J}$ Radiat Oncol Biol Phys. 2007; 67: 225-231.

34. Emami B, Lyman J, Brown A, et al. Tolerance of normal tissue to therapeutic irradiation. Int J Radiat Oncol Biol Phys. 1991; 21(1):109-122. 